\title{
CAMBIO DE APELLIDOS Y OTROS EFECTOS DERIVADOS DE LA DISOLUCIÓN DEL VÍNCULO MATRIMONIAL. COMENTARIO DE LA SENTENCIA DE LA AUDIENCIA PROVINCIAL DE GRANADA (SECCIÓN 5a) NÚM. 319/2017, DE 14 DE SEPTIEMBRE
}

\author{
CHANGE OF FAMILY NAMES AND OTHER EFFECTS \\ DERIVED FROM THE DISSOLUTION OF THE MARRIAGE. \\ COMMENT ON THE JUDGMENT OF THE SPANISH COURT \\ OF APPEAL OF GRANADA (SECTION 5) NUMBER 319/2017 \\ OF 14TH SEPTEMBER
}

\author{
Profa Dra. Carmen AzCÁRraga Monzonís \\ Profesor Titular de Derecho internacional privado \\ Universitat de València \\ Prof. Dr. Pablo Quinzá Redondo \\ Profesor Ayudante Doctor de Derecho internacional privado \\ Universitat de València
}

Recibido: 13.07.2018 / Aceptado: 24.07.2018

DOI: https://doi.org/10.20318/cdt.2018.4402

\begin{abstract}
Resumen: Cambio de apellidos de la esposa tras disolución del vínculo matrimonial, así como otros efectos derivados de la misma, no identificándose ni aplicándose correctamente la normativa de Derecho internacional privado aplicable el caso.

Palabras clave: cambio de apellidos, disolución del vínculo matrimonial, menores, alimentos, efectos patrimoniales del matrimonio.

Abstract: This case deals with the change of the family name of a wife after the dissolution of her marriage as well as other effects derived from that dissolution. The judgment seems not to identify or apply properly the Private international law rules governing the different matters involved in this case.

Keywords: change of family names, dissolution of marriage, minors, maintenance claims, matrimonial property regime.
\end{abstract}

Sumario: I. Introducción. II. Hechos. III. Cuestiones controvertidas. IV. La correcta fundamentación en normas de Derecho internacional privado. 1. La disolución del vínculo matrimonial. 2. Régimen económico matrimonial. 3. Alimentos. 4. Menores. 5. Cambio de apellidos. V. Valoración. 


\section{Introducción}

1. El 14 de septiembre de 2017, la Audiencia Provincial de Granada estimó un recurso de apelación en un asunto referente a un divorcio internacional en el que debía decidirse particularmente sobre la recuperación de los apellidos de la esposa antes de haber contraído matrimonio. Tras proporcionar los hechos (II), reflexionaremos sobre las cuestiones más controvertidas del fallo, partiendo de la premisa de que se trata de un asunto que presenta un elemento de extranjería (III). Posteriormente, toda vez que la resolución del caso no evidencia una correcta identificación y aplicación de la normativa de Derecho internacional privado que guarda relación con el objeto de la controversia, propondremos de manera prudente y razonada, la que consideramos habría sido una correcta fundamentación de los aspectos que conciernen al Derecho internacional privado (IV). Finalizaremos el comentario con unas conclusiones a modo de reflexión final (V).

\section{Hechos}

2. Con carácter previo a la descripción de los hechos es importante indicar que la sentencia objeto de comentario es extremadamente parca en lo referente a esta cuestión, encontrándose los elementos esenciales del caso únicamente concentrados en las cinco líneas del fundamento jurídico primero, que son a todas luces insuficientes. Ello nos ha obligado a incorporar una cierta dosis de presunción de hechos - por supuesto, razonada y coherente- ya que si de la lectura de la sentencia dependiera exclusivamente, difícilmente podría abordarse un comentario como el que estamos acometiendo.

3. Teniendo esto presente, los hechos pueden resumirse de la siguiente manera: el litigio enfrenta a dos cónyuges, ambos de nacionalidad rumana -lo que deducimos de la incorrecta aplicación del art. $107 \mathrm{Cc}$ al referirse a la ley de la nacionalidad común de los cónyuges- y con dos hijos, Enma y Eduardo, debiendo decidirse sobre los efectos personales y patrimoniales más relevantes de la disolución de su matrimonio. Se desconoce, a ciencia cierta, la residencia habitual de ambos cónyuges, aunque puede presumirse que hasta el año 2014 era España, momento en el que el marido decide abandonar nuestro país con destino incierto. Asumimos que la mujer, demandante, y los hijos seguían residiendo en España al tiempo de la presentación de la demanda, pues de lo contrario la resolución del caso podría ser bien distinta.

4. Partiendo de lo anterior, la sentencia de primera instancia, dictada el 13 de septiembre de 2016, parece centrarse, a tenor del extracto reproducido en la SAP de Granada, en la disolución por divorcio del matrimonio y en algunos de los efectos personales y patrimoniales de tal divorcio como son la protección de los hijos -incluyendo la patria potestad y los derechos de custodia y visita- y la pensión de alimentos. Contra dicha resolución presentó la demandante recurso de apelación referido exclusivamente a la posibilidad de recuperar los apellidos que tenía antes de haber contraído nupcias con el demandado, cuestión esta que da lugar a la SAP de Granada de 14 de septiembre de 2017.

\section{Cuestiones controvertidas}

5. Una vez descritos los hechos del caso, es turno de examinar las cuestiones jurídicas más controvertidas que, pese a ser varias, pueden resumirse en las dos siguientes: ausencia de aplicación (1) e incorrecta aplicación (2) de la normativa de Derecho internacional privado.

\section{Ausencia de aplicación de la normativa de Derecho internacional privado.}

6. Desde nuestro punto de vista, uno de los primeros errores que presenta la resolución es la no consideración de la normativa de Derecho internacional privado aplicable al caso concreto, lo que resulta claramente manifiesto en primera instancia, donde el juzgador asume que será el Cc español el 
aplicable a la patria potestad y los derechos de custodia y visita, así como a la pensión de alimentos, obviando -o al menos lo parece- que se trata de un asunto que presenta un elemento de extranjería -matrimonio entre dos sujetos de nacionalidad rumana con, al menos uno de ellos, residencia habitual en España- en el que primeramente debía haberse planteado si los tribunales españoles tenían competencia judicial internacional para resolver el caso y, seguidamente, la normativa a aplicar.

7. Dicha omisión se evidencia particularmente, en lo que respecta a la disolución del vínculo matrimonial así como en relación con la responsabilidad parental y las obligaciones de alimentos, echándose claramente de menos una referencia a la normativa de Derecho internacional privado que aborda tales cuestiones, como son el Reglamento (CE) n ${ }^{\circ}$ 2201/2003 del Consejo, de 27 de noviembre de 2003, relativo a la competencia, el reconocimiento y la ejecución de resoluciones judiciales en materia matrimonial y de responsabilidad parental (en adelante, Reglamento Bruselas II bis) ${ }^{1}$, el Reglamento (UE) no 1259/2010 del Consejo, de 20 de diciembre de 2010, por el que se establece una cooperación reforzada en el ámbito de la ley aplicable al divorcio y a la separación judicial (en adelante, Reglamento Roma III) ${ }^{2}$ y el Reglamento (CE) n ${ }^{\circ} 4 / 2009$ del Consejo, de 18 de diciembre de 2008, relativo a la competencia, la ley aplicable, el reconocimiento y la ejecución de las resoluciones y la cooperación en materia de obligaciones de alimentos

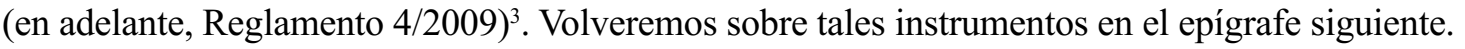

8. Sea como fuere, lo curioso de dicha ausencia es que únicamente tiene lugar en primera instancia, pues la SAP de Granada sí que evidencia que el caso a resolver es internacional: solo así se explica que se plantee la aplicación de una norma de conflicto - el art. $107 \mathrm{Cc}$-, aunque lo haga de manera incorrecta -como explicaremos más adelante-. Otra muestra de ello es que la segunda instancia también plantea la cuestión de la prueba del Derecho extranjero, una cuestión procesal que se presenta únicamente en casos internacionales.

9. En definitiva, puede afirmarse que la resolución presenta, en cierto modo, rasgos "bipolares" desde dos puntos de vista. Primero, porque el juzgador de primera instancia ha hecho caso omiso del carácter transfronterizo de la situación jurídica, cosa que no ha hecho el tribunal de apelación; segundo, porque desde la perspectiva de las cuestiones abordadas, algunas parecen haber sido tratadas como "internas" -patria potestad y alimentos-, mientras que otras sí han recibido explícitamente la consideración de "internacionales" -el cambio de apellidos-, aunque errando en la determinación de la norma de conflicto aplicable.

\section{Incorrecta aplicación de la normativa de Derecho internacional privado}

10. Como hemos indicado en el epígrafe anterior, la sentencia analizada pasa por alto la internacionalidad de la situación jurídica para las cuestiones planteadas en primera instancia -patria potestad y alimentos- pero sí plantea el Derecho aplicable para el cambio de apellidos de la esposa. Lo hace, sin embargo, invocando una norma de conflicto cuyo ámbito de aplicación es distinto. Aún es más, la SAP de Granada plantea la aplicación de una norma de conflicto que no resulta aplicable en la actualidad ni siquiera para la materia para la que está prevista. Veamos detenidamente ambas cuestiones, que aunque relacionadas entre sí, pueden deslindarse en dos errores distintos.

11. En primer lugar, el fundamento jurídico segundo de la sentencia menciona el art. $107 \mathrm{Cc}$, referente a la ley aplicable a la separación y el divorcio, para determinar la ley reguladora del cambio de apellidos. Por ello, la SAP Granada considera que al tener ambos sujetos nacionalidad rumana, el Dere-

${ }^{1}$ DO L 338 de 23 de diciembre de 2003. Nótese que dicho Reglamento podría ser próximamente modificado, si bien todo hace indicar que las normas de competencia judicial internacional en materia de crisis matrimoniales no sufrirán modificación relevante alguna (Propuesta de Reglamento del Consejo relativo a la competencia, el reconocimiento y la ejecución de resoluciones en materia matrimonial y de responsabilidad parental, y sobre la sustracción internacional de menores (refundición), COM (2016) 411).

2 DO L 343 de 29 de diciembre de 2010.

${ }^{3}$ DO L 7 de 10 de enero de 2009. 
cho aplicable es el rumano. Aquí encontramos el primer error. El art. $107 \mathrm{Cc}$ no es la norma de conflicto aplicable al cambio de apellidos, por más que éste sea solicitado por uno de los cónyuges en el marco de un procedimiento de divorcio. Dicha norma de conflicto se refiere estrictamente a la disolución del vínculo matrimonial por separación o divorcio, pero no a los efectos, bien personales o patrimoniales que pudieran derivarse del mismo.

12. Sin embargo, se une a dicho error que el tribunal de apelación invoca una norma de conflicto totalmente desfasada, tanto por existir un Reglamento de la Unión Europea aplicable en la materia, como por que su redacción fue modificada hace ya más de tres años. Así, la ley aplicable a la separación y el divorcio viene determinada por el Reglamento Roma III, cuya entrada en aplicación se produjo el 21 de junio de 2012. Junto a ello, conviene recordar que el punto de conexión del art. 107.2 Cc ya no es la nacionalidad común de los cónyuges, pues dicha norma de conflicto fue modificada por el legislador estatal en virtud del apartado veintiocho de la Disposición final primera de la Ley 15/2015, de 2 de julio, de la Jurisdicción Voluntaria, que establece que "La separación y el divorcio legal se regirán por las normas de la Unión Europea o españolas de Derecho internacional privado"-el citado Reglamento Roma III-.

13. Finalmente, merece una breve mención dentro de estas reflexiones la forma en que el tribunal resuelve la cuestión de la aplicación del Derecho extranjero, en esta ocasión el Derecho rumano. Señala la sentencia que el art. 281 LEC establece que el Derecho extranjero deberá ser objeto de prueba, en concreto su contenido y vigencia -como menciona también el art. 33.1 de la Ley 29/2015, de 30 de julio, de Cooperación Jurídica Internacional en Materia Civil, una normativa que es obviada en esta sentencia-. De acuerdo con el art. 282 LEC, la carga de la prueba corresponde a las partes, lo que deja al juez un papel secundario en el desempeño de esta labor a través de esos "medios de averiguación que estime necesarios" (art. 281.2 LEC). El juez debería desarrollar esta tarea únicamente como apoyo a las partes si se constata su diligencia en este contexto de conformidad con la jurisprudencia constitucional existente sobre la materia ${ }^{4}$. Llama pues la atención que se afirme que la carga probatoria corresponde a quien alega el Derecho de otro país, aunque "puede ser averiguado por el Juez". De igual modo, consideramos que la aportación por la parte de la mera "cita" de un precepto del Código civil rumano no debería haber sido suficiente para aplicarlo aunque no exista oposición sobre este extremo. Además, nada se dice sobre su vigencia ni los medios de prueba utilizados, pero, con todo, el tribunal lo considera "razonablemente acreditado" para confirmar la posibilidad de la demandante de recuperar su apellido.

\section{La correcta fundamentación en normas de Derecho internacional privado}

13. En el epígrafe anterior se ha puesto de manifiesto cómo no son pocos los errores cometidos, desde una perspectiva estrictamente internacionalprivatista, tanto por el juzgador de primera instancia, como por la SAP Granada. Así, la fundamentación del caso dista mucho de ser acertada en lo que respecta a la determinación y aplicación de las fuentes del Derecho internacional privado español. Es por ello que nos plantearemos en el presente epígrafe cuál habría sido, desde nuestro punto de vista, la fundamentación acertada desde la perspectiva internacionalprivatista, tomando en consideración los hechos expuestos y descartando los errores advertidos.

14. Teniendo presente que se trata de un supuesto referente a las crisis matrimoniales y sus efectos, distinguiremos tanto aquellas cuestiones que sí se tratan a lo largo de la sentencia -menores, alimentos y cambio de apellidos-, como aquellas que bien no se mencionan -régimen económico matrimonial- bien se referencian de una manera extremadamente breve -la disolución del matrimonio-. Para cada una de estas cuestiones nos plantearemos, primeramente, si los tribunales españoles tenían

${ }^{4}$ C. AzCÁrraga Monzonís, "Spain: The Application of Foreign Law in Spain - Critical Analysis of the Legal Novelties of 2015”, en NISHITANI, Y.: Treatment of Foreign Law - Dynamics Towards Convergence?, Springer, Heidelberg, 2017. 
competencia judicial internacional, y seguidamente, la ley aplicable. Nuestro propósito, lejos de explicar detalladamente la normativa de Derecho internacional privado y las concretas disposiciones aplicables, será identificar las mismas y plantear su aplicación práctica al asunto concreto.

\section{Disolución del vínculo matrimonial}

15. La primera cuestión relevante, de la que dependen las demás, se refiere a la disolución del vínculo matrimonial por divorcio, que fue precisamente el objeto de la demanda inicial de la esposa. Un divorcio que, a tenor de los hechos del caso, no planteó problema alguno, pues la sentencia parte del mismo para plantearse los consecuentes efectos personales y patrimoniales. No consideramos, sin embargo, acertada esta posición tan reduccionista: el tribunal de primera instancia debía haber examinado en primer lugar su competencia judicial internacional para determinar, a continuación, la ley reguladora de tal divorcio, operación que no tuvo lugar. Veamos, en este sentido, si los tribunales españoles eran competentes y qué ley resultaba ser la aplicable.

16. La competencia judicial internacional de los tribunales españoles para el divorcio no se plantea en ningún momento de la sentencia. No se encuentran referencias al Reglamento Bruselas II bis ${ }^{5}$ ni a la LOPJ. ¿Tenían realmente los tribunales españoles competencia judicial internacional? De acuerdo con la información facilitada en el caso, la respuesta debe ser afirmativa, toda vez que, la última residencia habitual común de los cónyuges se localizaba en España y la demandante continuaba residiendo en dicho Estado miembro al tiempo de la presentación de la demanda (art. 3.1.a) segundo inciso Reglamento Bruselas II bis). En definitiva, puede concluirse que el tribunal juzgador sí tiene competencia judicial internacional para resolver sobre la disolución del vínculo, si bien, como adelantábamos, a tenor de la información de la sentencia no consta que el tribunal llegara a plantearse tal cuestión.

17. Por otro lado, la ley aplicable al divorcio y la separación judicial viene regulada en el Reglamento Roma $\mathrm{III}^{6}$, un instrumento que no se menciona ni en primera ni en segunda instancia, ya que no se plantea en términos globales que la ley aplicable a la disolución del vínculo matrimonial pueda ser una distinta a la española. Asumiendo que los cónyuges no eligieron la ley aplicable (art. 5 Reglamento Roma III), el Derecho regulador vendrá determinado en virtud del art. 8 del Reglamento Roma III. Un análisis de las normas de conflicto de dicha disposición permite excluir la aplicación del apartado a) del Reglamento Roma III, ya que el demandado se marchó de España en 2014 y, asumimos que la demanda de divorcio y los efectos derivados del mismo se produjeron en un momento posterior, es decir, cuando ya no vivían juntos.

Menos certero resulta saber si la ley aplicable vendrá determinada por el apartado b) o c) del art. 8 del Reglamento Roma III. Si asumimos que la demanda se presentó en un plazo inferior a un año desde que los cónyuges finalizaron su residencia, entonces el Derecho aplicable será el español (apartado b). Si embargo, si la demanda se presentó posteriormente, corresponderá al Derecho rumano la regulación del divorcio (apartado c). Lamentablemente esta cuestión no se encuentra mencionada a lo largo de la SAP de Granada, en línea con lo que ocurre con la competencia judicial internacional.

\footnotetext{
5 Sobre el controvertido ámbito de aplicación personal del Reglamento Bruselas II bis, véase la sentencia del asunto del TJUE C-68/07 Kerstin Sundelind Lopez v Miguel Enrique Lopez Lizazo. Resulta asimismo interesante la lectura de J.RoDRÍGUEZ RoDRIGO, "Reglamento 1347/2000: ámbito de aplicación personal (arts. 7 y 8)", Revista colombiana de derecho internacional, núm., 4, 2005, pp. 361-378.

${ }^{6}$ Para una aproximación general a dicho Reglamento resultan interesantes, entre otros, los siguientes trabajos: CALVO CARavaca, A.L. y Carrascosa GonzÁlez, J.: "La ley aplicable al divorcio en Europa: el futuro Reglamento Roma III", Cuadernos de derecho transnacional, vol. 1, núm. 1, 2009, pp. 36-71; Palao Moreno, G. : "La ley aplicable al divorcio. El Reglamento (UE) núm. 1259/2010, de 20 de noviembre (Roma III)", en Heras Hernández, M. M. (Coord). y Peña Vicente, M. y Delgado Martín, P. (dir.): Nuevas orientaciones del derecho civil en Europa, Aranzadi, Cizur Menor, pp. 661-675 y Espinosa CALABUIG, R.: "El divorcio internacional en la Unión Europea: problemas de coherencia y coordinación normativa", Revista boliviana de derecho, núm. 22, 2016, pp. 208-233.
} 


\section{Régimen económico matrimonial}

18. Una vez analizada la disolución del vínculo matrimonial, es turno de plantearse uno de los efectos jurídicos más relevantes de la misma, como es la disolución y liquidación del régimen económico matrimonial. Llama la atención que, pese a que el fundamento jurídico primero de la SAP de Granada establece expresamente que la sentencia de primera instancia "acuerda el divorcio...con los efectos personales y patrimoniales inherentes", no se haga mención alguna al régimen económico matrimonial, pese a ser la distribución de los bienes de los cónyuges uno de los efectos económicos más importantes de la disolución del matrimonio. Esta ausencia, sin embargo, no obsta para que consideremos interesante plantearnos si, potencialmente, el tribunal español podría haber entrado a resolver tal cuestión y, de ser así, cuál habría sido la ley aplicable.

19. En primer lugar, las normas de competencia judicial internacional en materia de efectos del matrimonio -entre los que se encuentra el régimen económico matrimonial- se encuentran previstas en la LOPJ y en concreto en el art. 22. Así será al menos hasta el 29 de enero de 2019, momento en el que resultará de aplicación el Reglamento (UE) 2016/1103 del Consejo, de 24 de junio de 2016, por el que se establece una cooperación reforzada en el ámbito de la competencia, la ley aplicable, el reconocimiento y la ejecución de resoluciones en materia de regímenes económicos matrimoniales (en adelante, Reglamento 2016/1103) ${ }^{7}$.

20. El examen de los foros del art. 22 LOPJ permite concluir que, efectivamente, los tribunales españoles tenían competencia judicial internacional, no en virtud de los foros exclusivos -la materia matrimonial no lo es-, ni del foro de la autonomía de la voluntad -asumimos que no ha tenido lugar- ni tampoco del foro del domicilio del demandado -el demandado abandonó España en 2014- sino en aplicación del foro especial del art. 22 quáter c) LOPJ, que prácticamente transcribe literalmente -no sin errores y, por supuesto, sustituyendo la expresión "Estado miembro" por "España"- el art. 3 del Reglamento Bruselas II bis ${ }^{8}$. En particular, los tribunales españoles serían competentes porque fue en España donde el matrimonio tuvo su última residencia habitual y uno de los cónyuges -la demandante- todavía sigue residiendo en nuestro país.

21. En relación con la ley aplicable, y asumiendo que los cónyuges no celebraron pactos o capitulaciones matrimoniales, habrá que estar al art. $9.2 \mathrm{Cc}^{9}$ mientras no sea de aplicación el Reglamento 2016/1103. Como es bien sabido, el primer punto de conexión es la nacionalidad común de los cónyuges, por lo que siendo ambos cónyuges de nacionalidad rumana, la ley aplicable será la de Rumanía.

\section{Alimentos}

22. Las obligaciones de alimentos derivadas de la disolución del vínculo matrimonial y en relación con los hijos menores sí que se mencionan de manera expresa en la sentencia de primera instancia. Sin embargo, el juzgador considera directamente de aplicación los arts. 142 y ss. del Cc, sin plantearse siquiera la competencia judicial internacional y la ley aplicable, una carencia de referencias a la normativa de Derecho internacional privado que intentaremos paliar en las siguientes líneas.

23. En primer lugar, es importante recordar que las fuentes internacionales en materia de obligaciones de alimentos son, entre otras y por lo que interesa a este comentario, por un lado, el Reglamento (CE) $n^{\circ} 4 / 2009$ del Consejo, de 18 de diciembre de 2008, relativo a la competencia, la ley aplicable,

\footnotetext{
7 DO L 183/1-29 de 8 de julio de 2016.

8 C. Esplugues Mota/ J.L. Iglesias Buhigues/G.Palao Moreno,Derecho internacional privado, Tirant Lo Blanch, Valencia, $11^{\circ}$ ed., 2017 , pp. 461-463.

9 Un profundo estudio de dicha norma de conflicto, así como del art. $9.3 \mathrm{Cc}$, puede encontrarse en Diago Diago, M.P.: Pactos o capitulaciones matrimoniales en derecho internacional privado, Colección El Justicia de Aragón, Zaragoza, 1999 y E. Rodríguez Pineau, : Régimen económico matrimonial. Aspectos internacionales, Comares, Granada, 2002.
} 
el reconocimiento y la ejecución de las resoluciones y la cooperación en materia de obligaciones de alimentos (en adelante, Reglamento 4/2009) ${ }^{10} \mathrm{y}$, específicamente en relación con la ley aplicable, el Protocolo de 23 de noviembre de 2007 sobre la ley aplicable a las obligaciones alimenticias (en adelante, Protocolo de La Haya de 2007) ${ }^{11}$.

24. Comenzando por la competencia judicial internacional, y descartando el juego de la autonomía de la voluntad al haber menores de 18 años -aunque la edad de los hijos no la sabemos a ciencia cierta, la sentencia de primera instancia aplica un art. de Cc que habla de "menores no emancipados"(art. 4 Reglamento 4/2009), resulta evidente que habrá que estar a los foros contenidos en el art. 3 Reglamento 4/2009. Al menos tres foros de dicho artículo permiten aseverar la competencia judicial internacional de los tribunales españoles: el foro de la residencia habitual del acreedor de alimentos - la demandante reside en España- y los foros previstos en los apartados c) y d) de dicha disposición, referentes a la concentración de litigios en conflictos referentes a demandas accesorias al estado de las personas y a acciones relativas a la responsabilidad parental, respectivamente.

25. Por lo que respecta a la ley aplicable, su determinación dependerá de las normas de conflicto del Protocolo de La Haya de 2007, a las que remite el propio art. 15 del Reglamento 4/2009. En líneas generales, este instrumento prevé dos posibilidades: que las partes elijan la ley aplicable o que no lo hagan, en cuyo caso entrarán en juego las conexiones objetivas.

En el supuesto analizado puede descartarse la elección de la ley aplicable, ya que dicha posibilidad se encuentra prevista para litigios en los que no concurran menores de 18 años (art. 8.3 Protocolo de La Haya de 2007). Será, por tanto, la regla general la que establezca el Derecho regulador de las obligaciones de alimentos. Así, en virtud del art. 3 del Protocolo de La Haya de 2007, "Las obligaciones alimenticias se regirán por la ley del Estado de la residencia habitual del acreedor", lo que produce como resultado la aplicación de la ley española. Como es bien sabido, el Protocolo de La Haya de 2007 contiene una norma especial en favor de determinados acreedores - por ejemplo, obligaciones de alimentos de los padres a los hijos, como es el caso-, por lo que si la aplicación de la ley española no permitiera obtener los alimentos, podría recurrirse a la regla prevista en el art. 4 de dicho instrumento -en especial su apartado cuarto-.

\section{Menores}

26. La decisión analizada reproduce asimismo el fragmento de la sentencia recurrida relativo a los efectos del divorcio de las partes en sus dos hijos no emancipados. El Juzgado de primera instancia aplica el Derecho español a la patria potestad, la guardia y custodia y al régimen de visitas pero no proporciona información sobre su eventual competencia internacional para conocer de estas cuestiones ni el razonamiento que le ha conducido a aplicar los distintos preceptos del Código civil español en los que basa su decisión. Analicemos pues, primero, si los tribunales españoles eran realmente competentes, para confirmar después si el Derecho español es efectivamente el que debe regir las referidas cuestiones.

27. Por lo que respecta a la competencia de los tribunales españoles en materia de responsabilidad parental, esta cuestión queda regulada en el Derecho internacional privado español por el referido Reglamento Bruselas II bis, cuyo art. 1 establece que el mismo se aplicará tanto a las crisis matrimoniales mencionadas supra como "a la atribución, el ejercicio, la delegación, la restricción o la finalización

\footnotetext{
10 DO L 7 de 10 de enero de 2009.

${ }^{11}$ Una valoración de conjunto de las fuentes reguladoras de alimentos y los efectos derivados de su elevado número puede encontrarse en F.GARAU SOBRINO, "Las fuentes españolas en materia de obligaciones alimenticias: ¿hacia un derecho internacional privado extravagante?", Cuadernos de derecho transnacional, núm. 2, vol. 3, 2011, pp. 130-144. Igualmente, para una aproximación general del Reglamento, se recomienda Mª.A. Rodríguez VÁzQuez, "La regulación del Reglamento 4/2009 en materia de obligaciones de alimentos: competencia judicial internacional, ley aplicable y reconocimiento y ejecución de resoluciones", Revista electrónica de estudios internacionales, núm. 19, 2010, pp. 1-30.
} 
de la responsabilidad parental" ${ }^{\prime 2}$. Considerando pues de aplicación este instrumento de la Unión Europea, podemos afirmar que los tribunales españoles eran competentes para conocer de esta cuestión en virtud del art. 12.1 del Reglamento Bruselas II bis, según el cual los órganos jurisdiccionales del Estado miembro que conozcan sobre la disolución del matrimonio en aplicación del art. 3 tendrán competencia también en las cuestiones relativas a la responsabilidad parental vinculadas a dicha demanda si se dan los requisitos señalados, como ocurre en este supuesto. De este modo, siendo competentes los españoles para conocer del divorcio como se ha explicado en un apartado anterior, lo eran también consecuentemente para resolver sobre la responsabilidad parental relativa a los hijos.

28. El Reglamento Bruselas II bis se ciñe a dos de los sectores del Derecho internacional privado: la competencia judicial internacional, como terminamos de explicar, y el reconocimiento y ejecución de resoluciones de ámbito intracomunitario. Por lo tanto, al no regular la ley aplicable a la responsabilidad parental, será necesario acudir a otra fuente para determinar el Derecho estatal que ha de regir esta cuestión en el asunto que nos ocupa. La ley aplicable a la protección de menores se determinará de acuerdo con el Convenio de La Haya de 1996 relativo a la competencia, la ley aplicable, el reconocimiento, la ejecución y la cooperación en materia de responsabilidad parental y de medidas de protección de los niños ${ }^{13}$. Así lo indica el art. 9.6 Cc en redacción dada por la Ley 26/2015, de 28 de julio, de modificación del sistema de protección a la infancia y a la adolescencia, aunque su remisión resulte innecesaria.

El art. 15.1 del Convenio de La Haya de 1996 establece la aplicación de la ley de la autoridad que conoce del asunto (la llamada lex fori), lo que conduce a la aplicación del Derecho español en este supuesto en particular, al ser competentes los tribunales de nuestro país. Por lo tanto, nuestro Derecho ha sido correctamente aplicado a la responsabilidad parental en la sentencia objeto de análisis.

\section{Cambio de apellidos}

29. Finalmente, es importante plantearse también la regulación de la ley aplicable al cambio de apellidos, que constituye el objeto principal del recurso presentado en apelación por la demandante. Recordemos que esta ha sido una de las cuestiones que con poca fortuna se han abordado en la SAP de Granada, ya que en el fundamento jurídico segundo se ha indicado, con desacierto, que la ley aplicable a dicha cuestión será la determinada en virtud de la ley de la nacionalidad común de los cónyuges (según el anterior art. 107.2 Cc).

Así, en primer lugar conviene recordar que el nombre y los apellidos constituyen una cuestión que afecta directamente a la esfera íntima del individuo, formando parte por tanto del concepto de estatuto personal. En particular, España se encuentra vinculada en esta materia por tres Convenios internacionales: el Convenio relativo a la Ley aplicable a los nombres y apellidos, hecho en Múnich el 5 de septiembre de 1980; el Convenio relativo a los cambios de apellidos y de nombres, hecho en Estambul el 4 de septiembre de 1958 y el Convenio relativo a la expedición de un certificado de diversidad de apellidos, hecho en La Haya el 8 de septiembre de 1982.

30. Descartando directamente la aplicación de este último, dado que la demandante pretende recuperar su antiguo apellido y no tener ambos, queda por valorar los dos primeros. El Convenio de Estambul de 1958 podría resultar aplicable a priori pero excluye de su ámbito de aplicación los cambios de apellidos derivados de un cambio del estado civil de las personas (art. 1), como es el caso que se está

12 El art. 2.7 del Reglamento indica además qué debe entenderse por "responsabilidad parental" a los efectos de este instrumento, esto es, "los derechos y obligaciones conferidos a una persona física o jurídica en virtud de una resolución judicial, por ministerio de la ley o por un acuerdo con efectos jurídicos, en relación con la persona o los bienes de un menor. El término incluye, en particular, los derechos de custodia y visita". Nótese, nuevamente, la reforma en curso de este Reglamento, que en esta materia estudia M.A.Gandía SelLens, "El Régimen Europeo de responsabilidad parental: su funcionamiento y las principales propuestas de cambio a raíz de la modificación del Reglamento Bruselas II bis", La Ley Derecho de Familia: Revista jurídica sobre familia y menores, $\mathrm{N}^{\circ} .17,2018$.

13 Estudia la relación entre el Convenio de La Haya de 1996 y la normativa europea P.McEleavy, "The 1996 Hague Convention and the European Union: Connection and Disconnection", en Permanent Bureau of The Hague Conference on Private International Law (ed.): A Commitment to Private International Law. Essays in honour of Hans van Loon, Intersentia, 2013. 
analizando, en el que la demandante pretende recuperar su apellido anterior al haberse divorciado. Por lo tanto, aplicaremos en este asunto el Convenio de Múnich de 1980, de aplicación universal al amparo de su art. 2, lo que descarta la aplicación del art. $9.1 \mathrm{Cc}$, norma de conflicto de origen interno reguladora del estatuto personal.

El art. 1 del Convenio de Múnich conduce a aplicar la ley de la nacionalidad del sujeto, de ahí que, teniendo en cuenta que la demandante ostenta la nacionalidad rumana, corresponde al ordenamiento jurídico rumano determinar la posibilidad de cambio de apellido tras la disolución del vínculo matrimonial En la sentencia analizada se cita precisamente el Cc rumano para regular dicha cuestión aunque el "recorrido jurídico" de su aplicación es erróneo: insistimos en que el art. 107 Cc no regula la ley aplicable al cambio de apellidos. Por ello, es pura casualidad - si ambos cónyuges hubieran tenido distinta nacionalidad el resultado sería distinto- que se haya llegado a la aplicación del mismo Derecho que resultaría de aplicación si se hubiera considerado la norma de conflicto correcta.

Cuestión diferente es el tratamiento que ha sido dado a la aplicación de este Derecho extranjero, un tema que hemos tenido la oportunidad de analizar anteriormente. En definitiva, con base en la "razonable acreditación" del Derecho rumano - que desde luego dista mucho de ajustarse a lo exigido por la normativa reguladora de esta cuestión-, la AP de Granada estima el recurso presentado y acoge la pretensión de la demandante por la cual recupera su apellido.

\section{Valoración}

31. La SAP de Granada objeto de este comentario es sin duda un cúmulo de despropósitos desde el punto de vista internacionalprivatista. Así, se arrastran omisiones de la sentencia en primera instancia -la ausencia de consideración del elemento internacional y, por ende, la nula mención de la normativa de Derecho internacional privado aplicable al supuesto-, lo que da lugar a una fundamentación deficiente que, por desgracia, empeora en segunda instancia, al aplicarse una norma de conflicto -el art. $107 \mathrm{Cc}-$ para el cambio de apellidos que no solo no regula dicha cuestión, sino que además tiene en la actualidad una redacción distinta -como hemos explicado, ya no se hace mención a la nacionalidad común de los cónyuges, sino que se remite, aunque de manera indirecta, al Reglamento Roma III-.

32.Es por ello que con el presente comentario hemos intentado, en cierto modo, plantearnos cómo se debió haber fundamentado el caso en lo que respecta a cuestiones apriorísticas -no hemos entrado en el fondo, por exceder esta cuestión del ámbito de nuestra disciplina- como son la verificación de la competencia judicial internacional de los tribunales españoles y la potencial aplicación del Derecho español, que no se cuestiona (ni se confirma) en ningún momento en la sentencia analizada. Para ello, hemos identificado la normativa de Derecho internacional privado aplicable en cada materia y los concretos foros - para el caso de la competencia judicial internacional- y normas de conflicto -si hablamos de ley aplicable- que el juzgador debió haber tomado en consideración. 